\title{
Compression Digital Video Files using Singular Value Decomposition
}

\author{
Hasan Maher Ahmed \\ hasanmaher@uomosul.edu.iq \\ College of Computers Sciences and mathematics \\ University of Mosul, Mosul, Iraq
}

Received on: 25/03/2018

Accepted on: 27/08/2018

The development of data transfer technologies over multimedia or communications networks needs to be addressed to large storage units so that they can be sent and received in a packaged manner. It also takes a lot of time to transmit them while the current technologies are high standards, which reduces storage space and thus reduces transmission time and reception.

The aim of the study is to compress digital video files using SVD by inserting digital video and then analyzing it into a set of frames and then processing each frame using the compression algorithm used in the research. A set of measures were used to measure the quality of the compression.

The results showed that the use of a single value analysis technique was an effective method for compressing digital video files. After applying the work algorithms to a set of samples, the results also showed consistency and compatibility in the compression process with the samples that were treated.

Keywords: Digital Video Processing, Singular Value Decomposition (SVD), Data Compression.

$$
\begin{aligned}
& \text { كبس ملفات الفيديو الرقي باستخدام تقنية تحليل القيمة الأحادية } \\
& \text { حسن ماهر أحدد } \\
& \text { كلية علوم الحاسوب والرياضيات } \\
& \text { جامعة الدوصل، الدوصل، العرلق }
\end{aligned}
$$

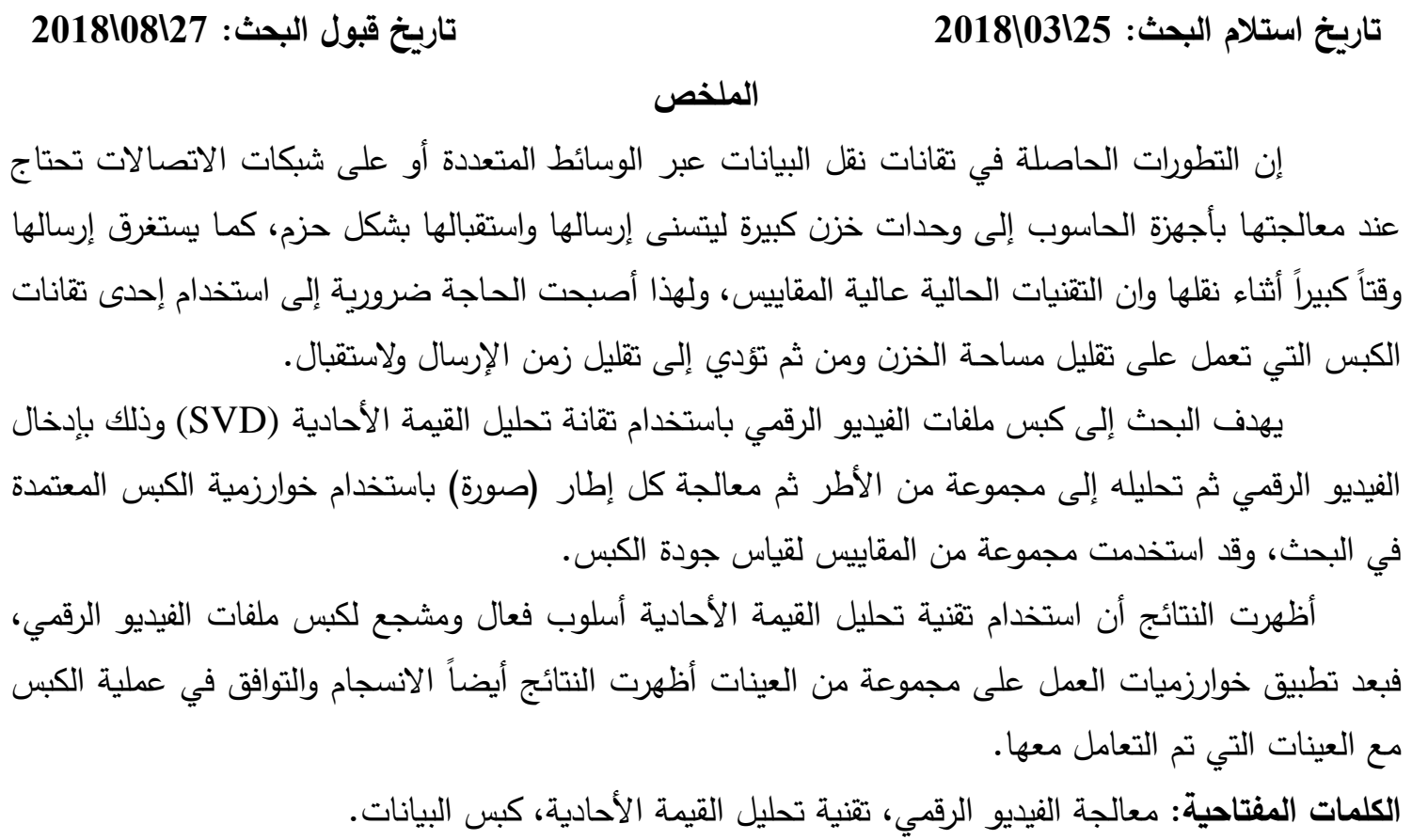


Introduction I المقدمة

في السنوات الأخيرة ازدادت ضخامة المعلومات والبيانات المخزونة التي تعالج رقمياً في مختلف الاختصاصات والتطبيقات بما فيها تطبيقات الاتصالات الحديثة؛ إذ يتطلب ارسال بيانات الفيديو الرقمي على نحو مباشر قناة ذاتيات التهات عرض حزمة واسعة، لهذا أصبحت عملية كبس البيانات وما زالت من التقانات المهمة في معالجة البيانات الرقمية

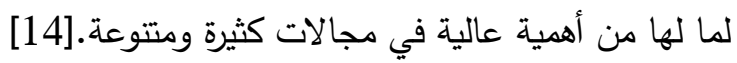
يعرف الكبس بأنه تقانة تستخدم لتقليل حجم ملفات الفيديو الرقمي الملون لغرض التبات التقليل من المساحة المخزنة

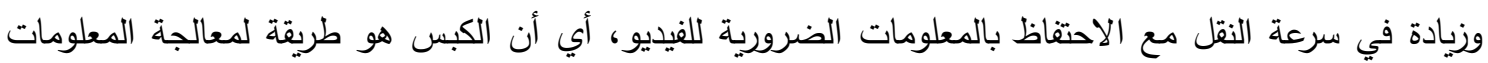

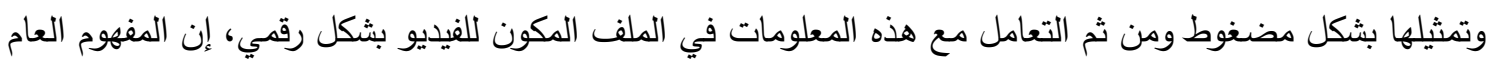
للكبس يأتي مع التعريف الدقيق للمعلومات الضرورية للوسائط المتعددة ولذلك يجب التمييز بين البيانات (data)

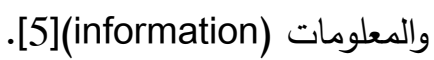
يتتاول البحث دراسة تقانة تحليل القيمة الأحادية Singular Value Decomposition (SVD) المشتقة من موضوع الجبر الخطي؛ إذ تعد هذه التقانة من التقانات التي لها تطبيقات عديدة ومختلفة وإحدى هذه المجالات التي يتتاولها البحث هو استخدامها في موضوع معالجة بيانات الفيديو الرقمي وذلك بتطبيق هذه التقانة لكبس الفيديو

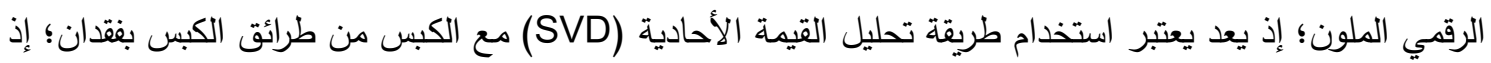

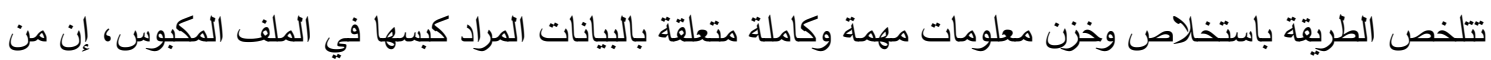

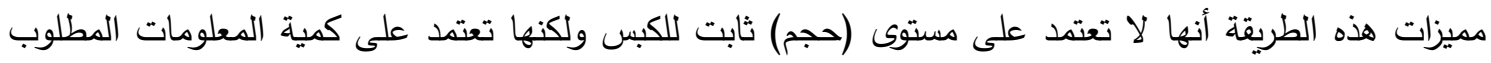

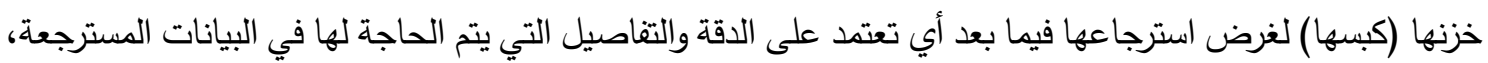

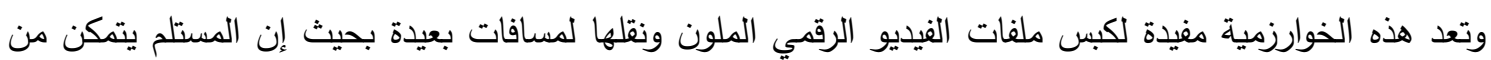

$$
\text { استرجاع البيانات بشكل مقبول. }
$$

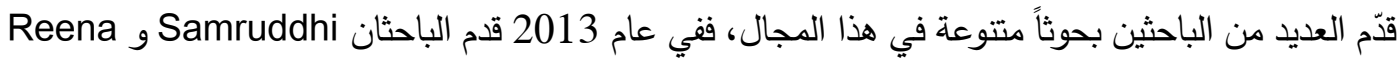
بحثاً تم فيه كبس الصور الرقمية الرمادية باستخدام تقانة تحليل القيمة الأحادية، واعتمد الباحثان على تقسيم الصورة

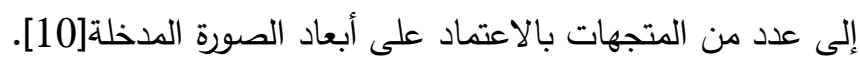
وفي عام 2016 قدم الباحثون Aishwarya و Rachana و Sipula Sobarad ونثاً تم فيه تطبيق تقانة تحليل القيمة الأحادية (SVD) على الصور الرقمية المتوسطة المستوى وذلك بعملية التحويل إلى ثلاث مصفوفات للصورة الرقمية الملونة [1]. وفي عام 2017 قدم الباحثان Maytham و بحثاً تم فيه الاعتماد على طريقة مهجنة بين تقانة تحليل القيمة الأحادية وتقانة التحويل المويجي المتقطع لكبس مقطع صوتي للكلام وذلك من خلال تسلسل لمجموعة من الخطوات باستخدام مرشح التمرير العالي لإزالة مقاطع الصمت واستخدام طريقة ترميز هوفمان [6].

\section{2. المخطط العام للبحث General Research Plan}

إن الهدف من البحث هو كبس ملفات الفيديو الرقمي الملون باستخدام تقانة تحليل القيمة الأحادية (SVD) وذلك بإخخال ملف الفيديو الرقمي الملون ثم تقسيمه إلى مجموعة من الأطر (Frames) ليتم بعد ذلك معالجة كل التل

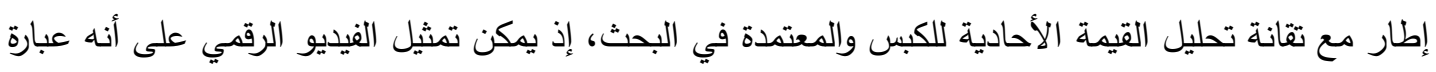


عن سلسلة من الصور الساكنة المسماة بالأطر (frames) إذ تعرض بالترتيب وحسب سرعة معينة؛ إذ إن الملفات المعتمدة لتمثيل حركة الفيديو هي عبارة عن ملفات ذات صيغ مختلفة تحمل بيانات بطرائق مختلفة؛ إذ تتفصل هذه الصور إلى أطر (Frames) كل واحدة منها تمثل لقطة من المشهد، ومن الممكن أن يكون حجم الملف كبيراً

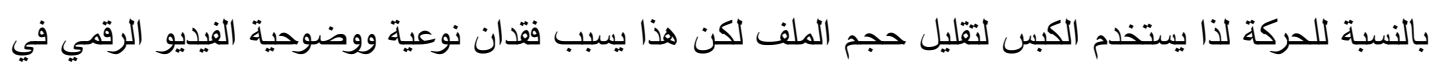

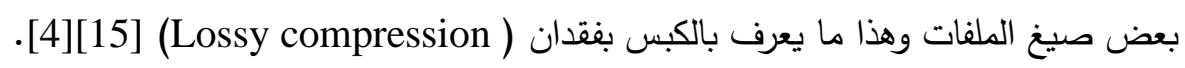
إن الخصائص المشتركة لأطر الفيديو هي أن النقاط المتجاورة تكون مترابطة فيما بينها لذلك يحتوي كل إطار على معلومات متكررة على نحو كبير والفعالية المهمة في ذلك هي إيجاد تمثيل جديد لتلك النقاط بأسلوب

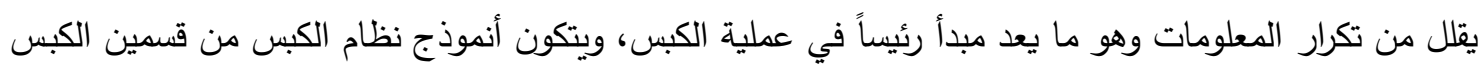

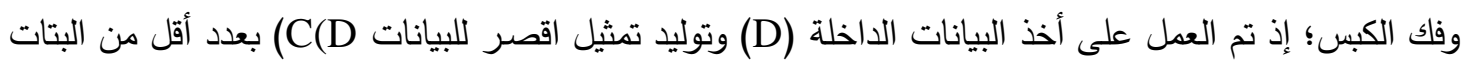

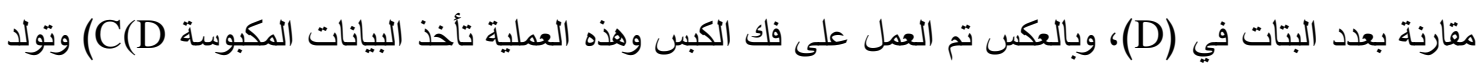

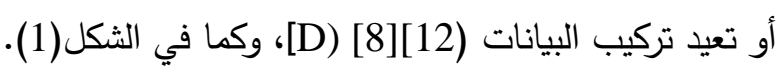

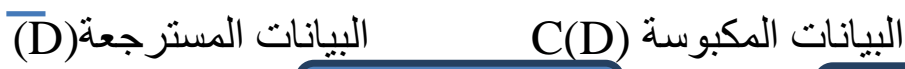

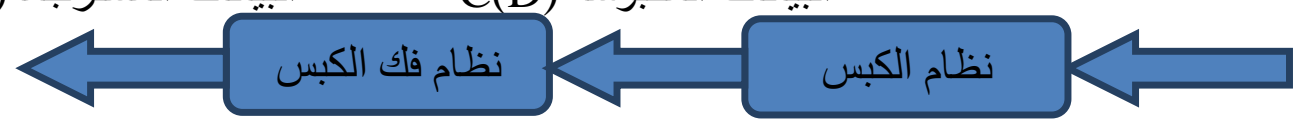
الثكل(1) مرحلة الكبس ومرحلة فلك الكبس

بعد إدخال الفيديو الرقمي الملون قسم إلى الشرائح اللونية الثلاثة (الأحمر والأخضر والأزرق)، وبالاعتماد على تقانة تحليل القيمة الأحادية (SVD) عولجت كل شريحة لونية على حدة، وذلك بدراسة القيم اللونية الخاصة بكل

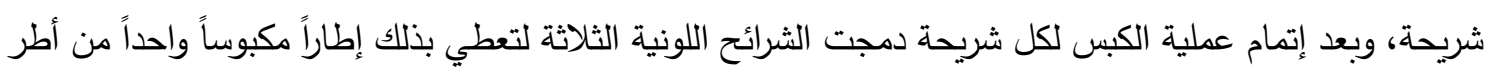
الفيديو، وبتكرار هذه العملية على جميع أطر الفيديو تم الحصول على ملف فيديو رقمي مكبوس وكما موضح في المخطط (1).

3. تقانة تحليل القيمة الأحادية Singular Value Decomposition (SVD)

تعد تقانة تحليل القيمة الأحادية من التقانات التي لها تطبيقات مختلفة وإحدى هذه المجالات هو استخدامها في كبس المعلومات لتقليل سعة الخزن ومن ثم زيادة سرعة النقل، إذ تتلخص هذه الطريقة بتحليل (تفكيك) المصفوفة التي تحوي بيانات أطر الفيديو الرقمي إلى ثلاث مصفوفات وتستخلص المعلومات المطلوبة من هذه المصفوفات لتكبس ويتم الحصول على أطر ذات تفاصيل يمكن أن تكون قريبة من أطر الفيديو الأصلي ولكن بحجم أقل؛ ويمكن

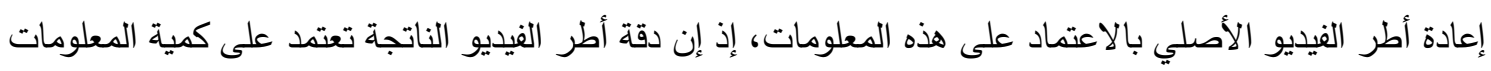

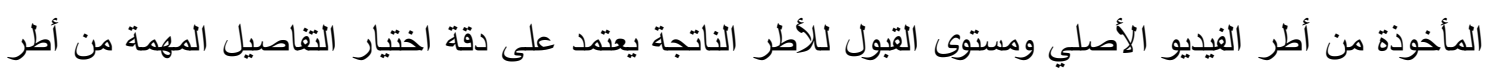

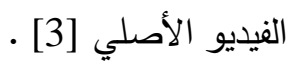
إن هذه التقانة هي ليست عملية كبس اعتيادية على الفيديو الرقمي فهي تقانة تتطلب عمليات رياضية عالية

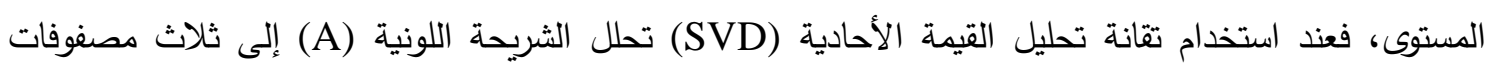
U,S,VT $A_{m \times n}=U_{m \times m} S_{m \times n} V_{n \times n}^{\top}$ 


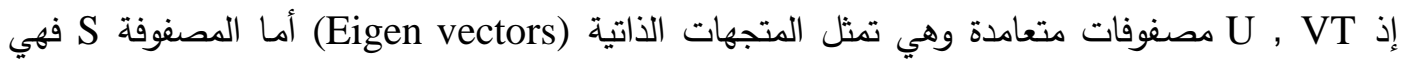
مصفوفة قطرية تمثل القيمة الذاتية (Eigen value)، فالمصفوفة U تضم القيم الموجودة في الجهة اليسرى من المصفوفة A، أما المصفوفة V فتضم القيم الموجودة في الجهة اليمنى من المصفوفة A، والمصفوفة S S تضم

عناصر القطر الرئيس للمصفوفة A، وتكون عناصر المصفوفة S مرتبة تتازلياً على القطر الرئيس؛ [2] إذ 0 $\left(\begin{array}{llll}\sigma_{1} & 0 & 0 & 0 \\ 0 & \sigma_{2} & 0 & 0 \\ 0 & 0 & \sigma_{\mathrm{N}} & 0 \\ 0 & 0 & 0 & 0\end{array}\right)\left(\mathrm{A}^{\mathrm{T}} \mathrm{A}-\lambda \mathrm{I}\right) \mathrm{X}=0$

\section{البداية}

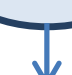

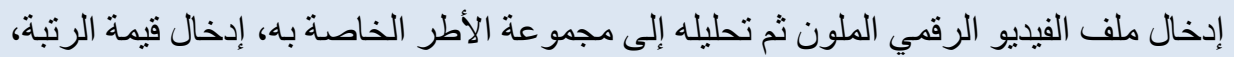

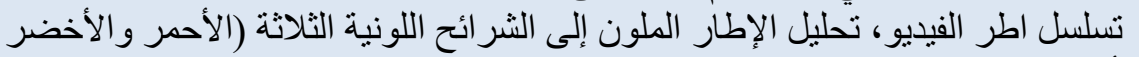

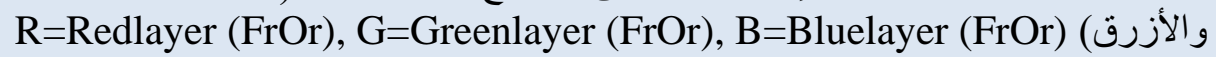

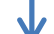

حساب القيم الذاتية للمصفوفة (S) و المتجهات الذاتية للمصفوفتين (U, V) ثم استخلاص المعلومات المطلوبة باستخدام قيمة الرتبة المبن

U=U* ديث(U,V) بالقيم الذاتية للمصفوفتين S دمبن الجذر التربيعي للمصفوفة

$$
\begin{aligned}
& \text { ، } \mathrm{V}=\mathrm{V} * \sqrt{s}, \sqrt{s} \\
& \text { خزن المصفوفات (U,V) كبيانات مكبوسة }
\end{aligned}
$$

دمج الثر ائح اللونبة الثلاثة الناتجة لتكوين الإطار الملون المكبوس و إضافته إلى لى ملف الفيديو المكبوس الاطن النمور

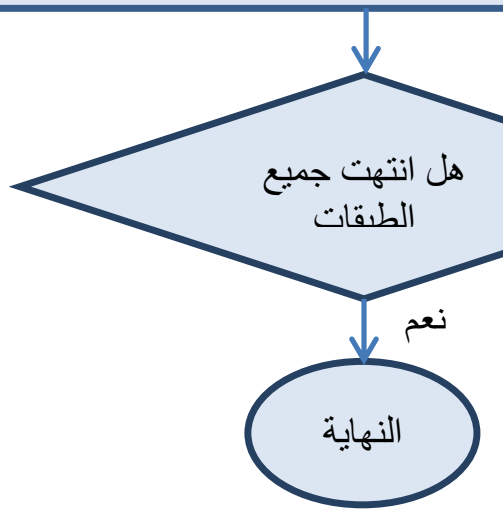

المخطط الانسيابي (1) خوارزمية الكبس باستخدام تقانة تحليل القيمة الأحادية 


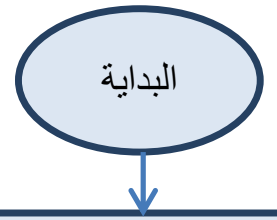

إدخال ملف الفيديو الرقمي المكبوس ثم تحليله إلى مجمو عة الأطر الخاصة به، ثم إدخال اسم الإطار

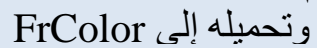

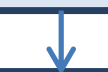

تحليل الإطار الملون إلى الثرائح اللونية الثلاثة (الأحمر والأخضر والأزرق)

G=Greenlayer (FrColor), B=Bluelayer R=Redlayer (FrColor),

(FrColor)

استرجاع الطبقات أو المستويات بعد الكبس عن طريق حساب المعادلات لكل مسنوى

$$
\begin{gathered}
\text { GFrColor }=U_{G} * V_{G}^{T} \quad \text { RFrColor }=U_{R} * V_{R}^{T} \\
\text { BFrCoLor }=I I_{D}^{*} V_{D}^{T}
\end{gathered}
$$

دمج الثرائح اللونية الثلاثة الناتجة لتكوين الإطار الملون بعد فلك الكبس، ثم إضافة الإطار الملون الناتج إلى ملف الفيديو الناتج

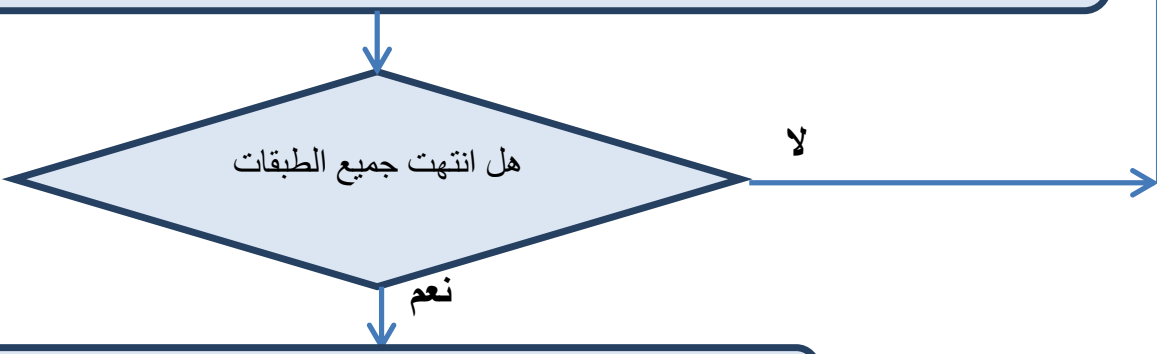

حساب قيم MSNR و MSE وحجم البيانات المكبوسة ونسبة

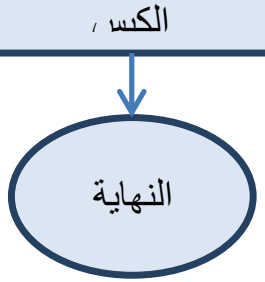

المخطط الانسيابي (2) خوارزمية فك الكبس باستخدام تقنية تحليل القيمة الأحادية

يتطلب حساب المصفوفات U,S,V'V وتكوينها العمليات الحسابية الموضحة في الخطوة التالية التي تتطلب

إيجاد قيم المتجه الذاتي والقيمة الذاتية، أما خطوات عمل الخوارزمية فيكون بالثكل الاتي:[7] 1-تحسب القيم الذاتية لكل من

$(\mathrm{A}-\mathrm{K} \mathrm{I})=0$

إذ إن A تمثل AA و A A و Aإن א تمثل القيم الذاتية، و ا تمثل مصفوفة قطرية عناصر قطرها يساوي 
2-تحسب المتجهات الذاتية بالاعتماد على القيمة الناتجة من النقطة الأولى لكل من AA و A'A من العلاقة (إذ

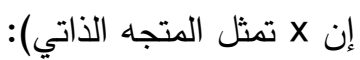
$(A-K I) x=0$

3-بعد إيجاد القيم الذاتية تكون المصفوفات الثلاثة U,S,V وعلى النحو الآتي: ا. تكوين عناصر المصفوفة الأحادية S بأخذ الجذر التربيعي لكل عنصر من عناصر القيم الذاتية مرتبة تنازلياً.

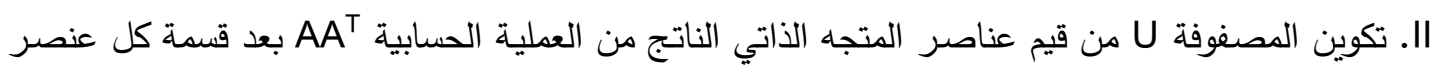

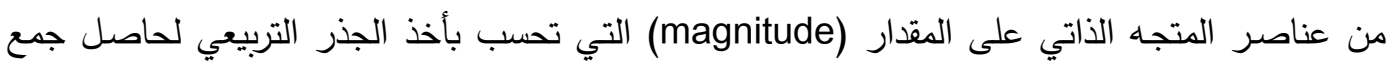
مربعات قيم المتجه الذاتي. III. أما عناصر المتجه الذاتي الناتجة من العملية الحسابية A'A فتتكون منها عناصر المصفوفة V بعد قسمة كل عنصر بالمتجه على قيمة المقدار (magnitude) للمتجه.

$\mathrm{A}=\mathrm{U} * \mathrm{~S} * \mathrm{~V}^{\top}$ 4-عند استرجاع المصفوفة A يتم ضرب المصفوفات الثلاثة:

عند تتفيذ الخوارزمية يتم إدخال قيمة الرتبة (Rank) التي تعتمد عليها نسبة الكبس ثم تطبيق خوارزمية

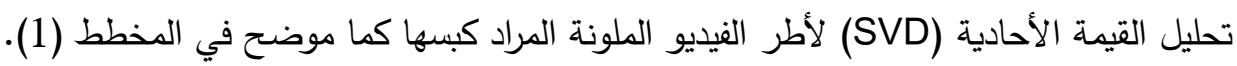

\section{4. أنموذج نظام كبس الفيديو الرقمي Digital Video Compression System}

إن الكبس يمكن أن ينجز بعدة طرائق وأساليب تعتمد على نوعية البيانات المراد إجراء الكبس عليها وعلى وفق

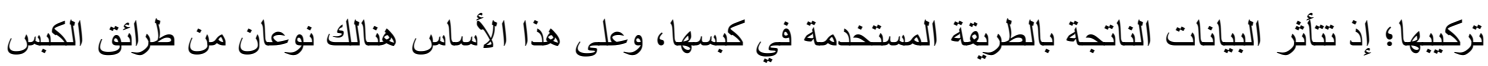

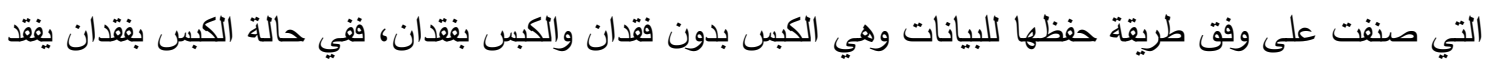

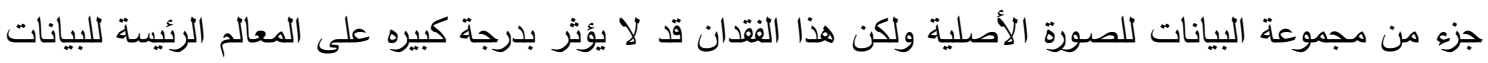

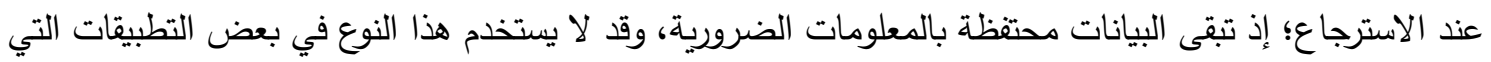
لا تسمح بأي فقدان في بياناتها مثل تطبيقات الوسائط المتعددة الطبيعية والبيانات النصية فيكون هذا النوع قليل الاستخدام، في حين يمكن استخدامه في تطبيقات التحس النائي بسبب كبر حجم المعلومات المرسلة، إن أهم ميزات هذا الأسلوب هو الحصول على نسبة كبس عالية وعليه يكون استخدامه بالاعتماد على نسبة فقدان البيانات المسموح

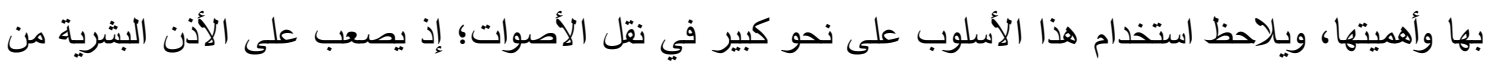
التمييز بين الصوت قبل الكبس والصوت بعد استرجاعه. بعد إدخال ملف الفيديو الرقمي الملون يقطع إلى مجموعة من الأطر المكونة منه، ثم تحلل كل إطار إلى لى إنى الثرائح اللونية الثلاثة (الأحمر والأخضر والأزرق) وكما في الثكل (2)، ثم تحلل كل شريحة إلى القيم الثيم اللونية الخاصة بها لتعالج بإدخالها إلى خوارزمية تحليل القيمة الأحادية. 


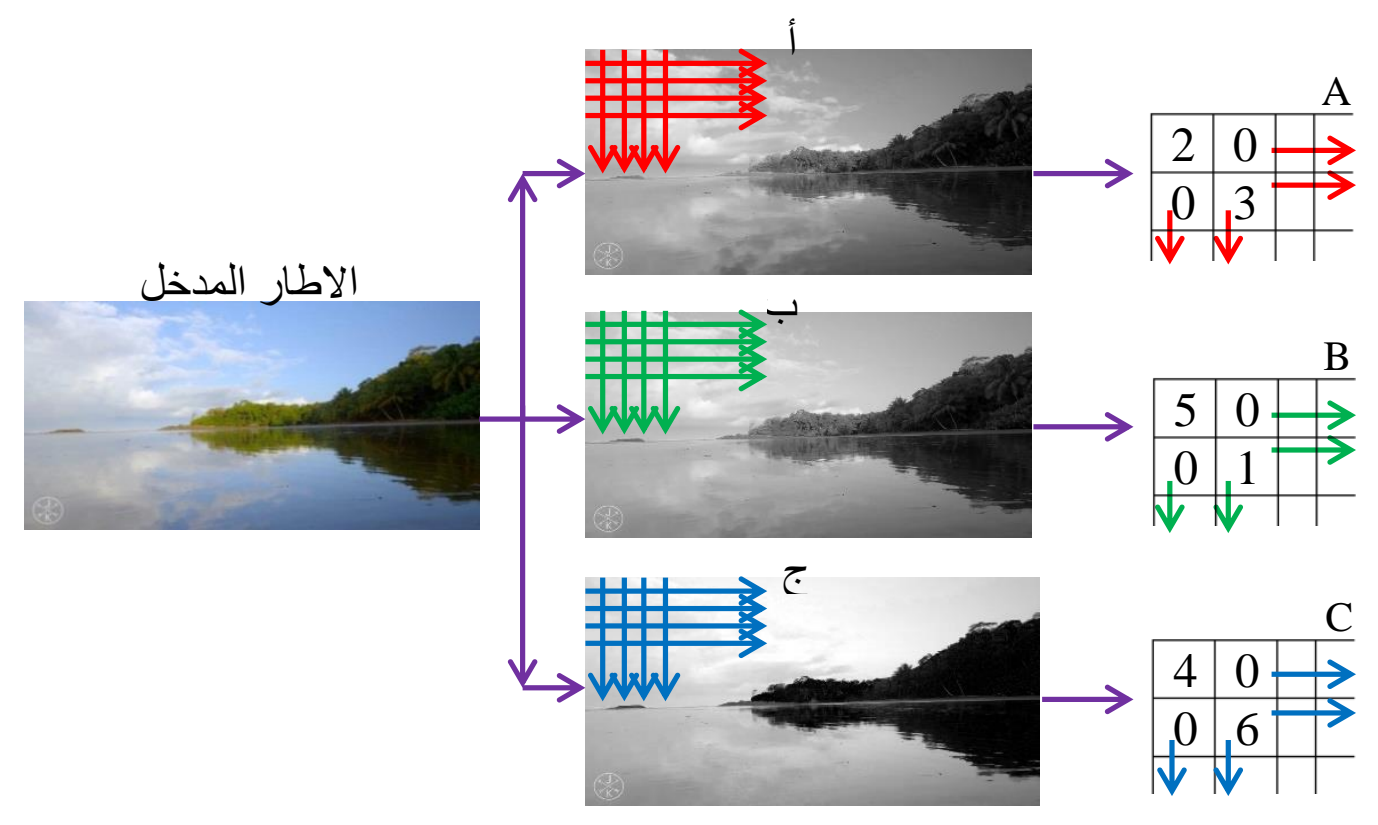

الثكل (2) تحليل إلى الشرائح اللونية: أ-الشريحة الحمراء، ب-الشريحة الخضراء، ج-الشريحة الزرقاء ونأخذ مثالاً على ذلك بأخذ عينة بيانات من إحدى أطر الفيديو المدخل بالاعتماد على الثريحة الحمراء

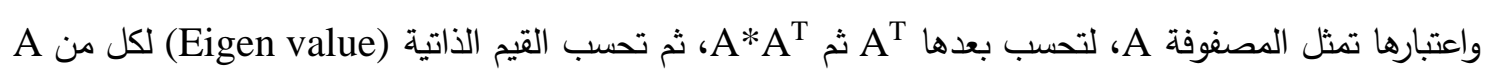
A وعلى النحو الاتي:

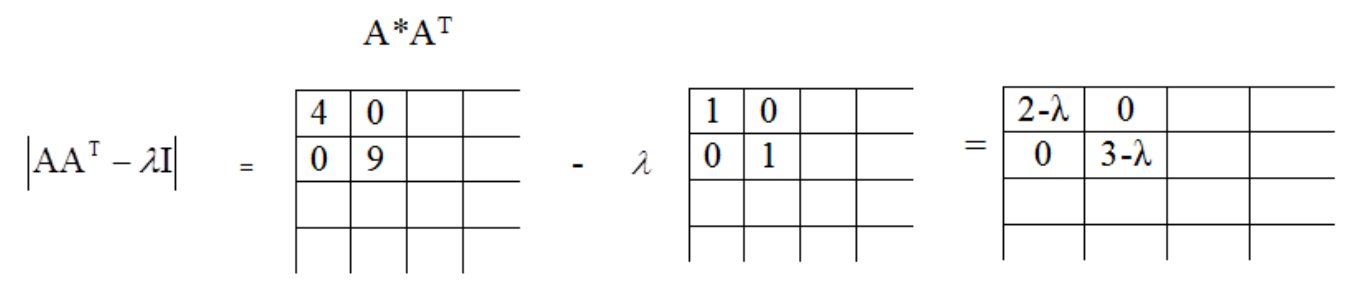

$(4-\lambda)(9-\lambda)-0=0, \quad(4-\lambda)(9-\lambda)=0, \quad \lambda_{1}=4, \lambda_{2}=9$

إذ التربيعي للقيم الذاتية (Eigen value) وترتيبها تنازلياً: $S=\left[\begin{array}{cc}\sqrt{4} & 0 \\ 0 & \sqrt{9}\end{array}\right]$

ثم تحسب قيم المتجهات الذاتية لكل من AA A $^{\mathrm{T}} \mathrm{A}$ و بعدما تم إيجاد القيم الذاتية لكليهما وعن طريق

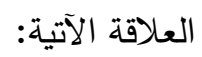

$$
\left(\mathrm{AA}^{\mathrm{T}}-\lambda \mathrm{I}\right) \mathrm{X}=0 \longmapsto\left(\left[\begin{array}{ll}
4 & 0 \\
0 & 9
\end{array}\right]-\lambda\left[\begin{array}{ll}
1 & 0 \\
0 & 1
\end{array}\right]\right) \mathrm{X}=0 \Longrightarrow\left[\begin{array}{cc}
4-\lambda & 0 \\
0 & 9-\lambda
\end{array}\right] \mathrm{X}_{1}=0
$$




$$
\begin{aligned}
& \lambda=4 \Longrightarrow\left[\begin{array}{cc}
4-4 & 0 \\
0 & 9-4
\end{array}\right] \mathrm{X}_{1}=0 \Longrightarrow\left[\begin{array}{ll}
0 & 0 \\
0 & 5
\end{array}\right] \mathrm{X}_{1}=0 \quad \longrightarrow \mathrm{X}_{1}=\left[\begin{array}{c}
-1 \\
0
\end{array}\right] \\
& \lambda=9 \Longrightarrow\left[\begin{array}{cc}
4-9 & 0 \\
0 & 9-9
\end{array}\right] X_{2}=0 \Longrightarrow\left[\begin{array}{rr}
-5 & 0 \\
0 & 0
\end{array}\right] X_{2}=0 \Longrightarrow X_{2}=\left[\begin{array}{l}
0 \\
1
\end{array}\right]
\end{aligned}
$$

بعدها يتم العمل على تكوين المصفوفة Uن من عناصر قيم المتجهات الذاتية x1,x2 بعد تقسيمها على قيم

(Magnitude). المقدار

$$
\begin{aligned}
& U=\left[\begin{array}{ll}
-1 / \sqrt{1} & 0 \\
0 & 1 / \sqrt{1}
\end{array}\right] \\
& \left(\mathrm{A}^{\mathrm{T}} \mathrm{A}-\lambda \mathrm{I}\right) \mathrm{X}=0 \Longrightarrow\left(\left[\begin{array}{ll}
4 & 0 \\
0 & 9
\end{array}\right]-\lambda\left[\begin{array}{ll}
1 & 0 \\
0 & 1
\end{array}\right]\right) \mathrm{X}=0 \longrightarrow\left[\begin{array}{cc}
4-\lambda & 0 \\
0 & 9-\lambda
\end{array}\right] \mathrm{X}=0 \\
& \lambda=4 \Longrightarrow\left[\begin{array}{cc}
4-4 & 0 \\
0 & 9-4
\end{array}\right] \mathrm{X}_{1}=0 \Longrightarrow\left[\begin{array}{cc}
0 & 0 \\
0 & 5
\end{array}\right] \mathrm{X}_{1}=0 \Longrightarrow \mathrm{X}_{1}=\left[\begin{array}{c}
-1 \\
0
\end{array}\right] \\
& \lambda=9 \Longrightarrow\left[\begin{array}{cc}
4-9 & 0 \\
0 & 9-9
\end{array}\right] X_{2}=0 \Longrightarrow\left[\begin{array}{cc}
-5 & 0 \\
0 & 0
\end{array}\right] X_{2}=0 \Longrightarrow X_{2}=\left[\begin{array}{l}
0 \\
1
\end{array}\right]
\end{aligned}
$$

بعدها يتم العمل على تكوين المصفوفة V من عناصر قيم المتجهات الذاتية X و X2 بعد تقسيمها على قيم

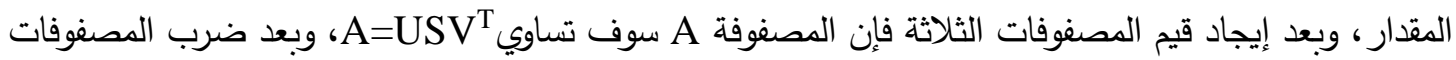
الثلاثة يتم الحصول على المصفوفة الأصلية A التي تساوي:

$$
V=\left[\begin{array}{ll}
-1 / \sqrt{1} & 0 \\
0 & 1 / \sqrt{1}
\end{array}\right] \Longrightarrow A=\left[\begin{array}{cc}
-1 & 0 \\
0 & 1
\end{array}\right]\left[\begin{array}{cc}
\sqrt{4} & 0 \\
0 & \sqrt{9}
\end{array}\right]\left[\begin{array}{cc}
-1 & 0 \\
0 & 1
\end{array}\right] \Longrightarrow \mathrm{A}=\left[\begin{array}{cc}
2 & 0 \\
0 & 3
\end{array}\right]
$$

عند استخدام تقانة تحليل القيمة الأحادية للكبس فإن هنالك مشكلة رئيسة وهي البدء بمصفوفة N*M وعند تطبيق هذه التقانة عليها سوف تتكون ثلاث مصفوفات بالأبعاد N*M و N*N والمصفوفة القطرية N"M، وإذا كانت M=N أي إن المصفوفة مربعة فتكون الصورة تمتلك القيم وبعد تطبيق تقانة تحليل القيمة الأحادية عليها سيتم الحصول على 2M²+M من القيم لكل تمثيل للأطر ، وهذا يتطلب مساحة خزن أكبر من المساحة المطلوبة للأطر الأصلية ولغرض تقليل مساحة الخزن واستخدام المعلومات الناتجة من طريقة تحليل القيمة الأحادية في عملية الكبس تطبق عملية التقريب (Upproximation) أي عملية ترتيب لعدد عناصر المصفوفات الناتجة (U,S,V) وذلك بتطبيق ما يعرف بال RANK أي الرتبة وهذا يتم عن طريق خزن جزء من عناصر المصفوفات الثلاث أي

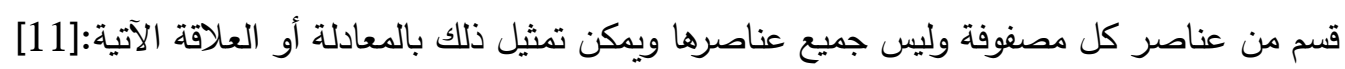
$\tilde{\mathrm{A}} \sum_{i=1}^{r} u i * s i * v i$ $\tilde{\mathrm{A}}=\sum_{i=1}^{r} u i * v i^{\mathrm{T}} \longrightarrow u i=\sqrt{s i} * u i \Rightarrow v i=\sqrt{s i} * v i$ إذ إن r تمثل قيمة الرتبة التي تمثل كمية المعلومات المطلوب خزنها ومقدارها أي (المعلومات المأخوذة من كل إطار)، وإن قيمة الرتبة تعتمد على المستخدم؛ إذ كلما كان مقدار الرتبة كبيراً كانت مساحة الخزن للمعلومات 
كبيرة ولكن دقة الفيديو وشدة الوضوح تقترب من الفيديو الأصلي، وكلما قل مقدار قيمة الرتبة قلت مساحة الخزن

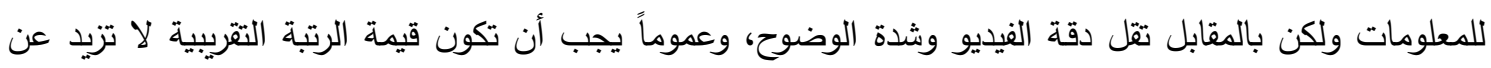
القيمة الناتجة من العلاقة (M/(2M+1 لكي يخزن الفيديو على نحو ملائم من حيث المساحة ودقة وضوح الفيديو وتكون نسبة الكبس مقبولة، ويمكن أيضاً التقليل من مساحة الخزن وذلك بدمج وتضمين عناصر المصفوفة القطرية S و ل وذلك بأخذ قيمة الجذر التربيعي لكل عنصر من عناصر المصفوفة S V وضربه

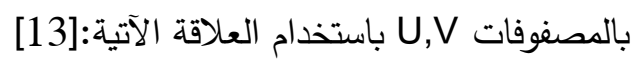
إن المساحة الخزنية لتمثيل المعلومات تقل وذلك بخزن كل من قيم المصفوفات U و V فقطط؛ لأن قيم

المصفوفة S أصبحت ضمن U و V وكذلك عدد العناصر المخزونة تعتد على قيمة الرتبة. measures of accuracy \& efficiency مقاييس كفاءة عملية الكبس الموضوعية

في بعض أنظمة إرسال واستقبال الملتيميديا يمكن التسامح ببعض الأخطاء في البيانات التي أعيد بناؤها،

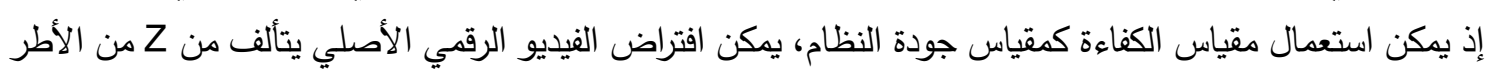

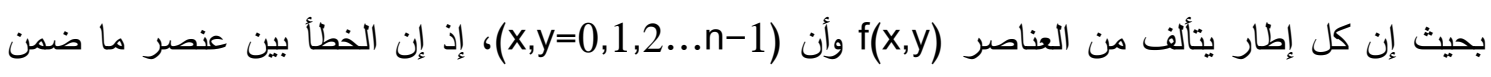

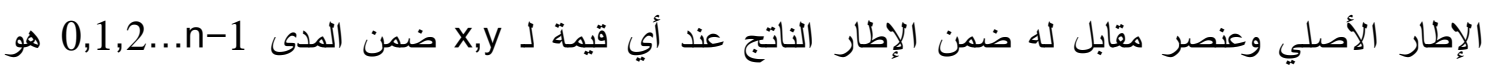
$e(x, y)=g(x, y)-f(x, y)$

بعد أن طبقت جميع خطوات العمل والحصول على نتائجها، تم تقييم النتائج النهائية بوساطة الاختبارات الإحصائية؛ إذ إن الاختبارات الإحصائية تستخدم طرائق رياضية، وتوجد عدة أنواع من الاختبارات الإحصائية أهمها:

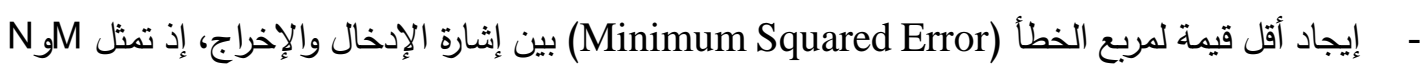

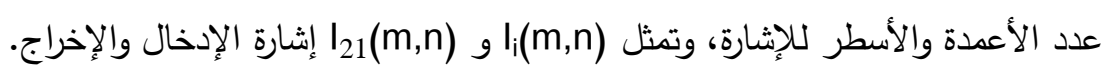

$\operatorname{MSE}=\left(\sum_{M N}\left[I_{i}(m, n)-I_{21}(m, n)\right]^{2}\right) /\left(M^{*} N\right)$

$\mathrm{PSNR}=$

-

$10 \log _{10}\left[\mathrm{R}^{2} / \mathrm{MSE}\right]$

إذ تمثل Rloating Point قيم البيانات إذا كانت Florigned integer أو - حساب نسبة الكبس وهي عبارة عن قسمة حجم الملف غير المكبوس على الملف المكبوس.

Compression ratio $(\mathrm{CR})=$ uncompressed file size / compressed file size

$$
\text { طبقت مقاييس الكفاءة على مجموعة عينات (أطر) مختلفة من الفيديو الرقمي والحصول على النتائج وكما في }
$$

\begin{tabular}{|c|c|c|c|}
\hline Frame No. & MSE & PSNR & CR \\
\hline 1 & 134.2482 & 26.8517 & 1.4750 \\
\hline 10 & 136.9313 & 26.7658 & 1.4500 \\
\hline 15 & 276.5838 & 23.7125 & 1.3000 \\
\hline 23 & 203.3147 & 25.0491 & 1.7179 \\
\hline 30 & 861.8283 & 18.7766 & 1.9385 \\
\hline 37 & 174.5117 & 25.7126 & 1.1351 \\
\hline 45 & 160.1511 & 26.0855 & 1.3500 \\
\hline
\end{tabular}


الجدول (1): تطبيق مقاييس الكفاءة على مجمو عة مختلفة من الأطر
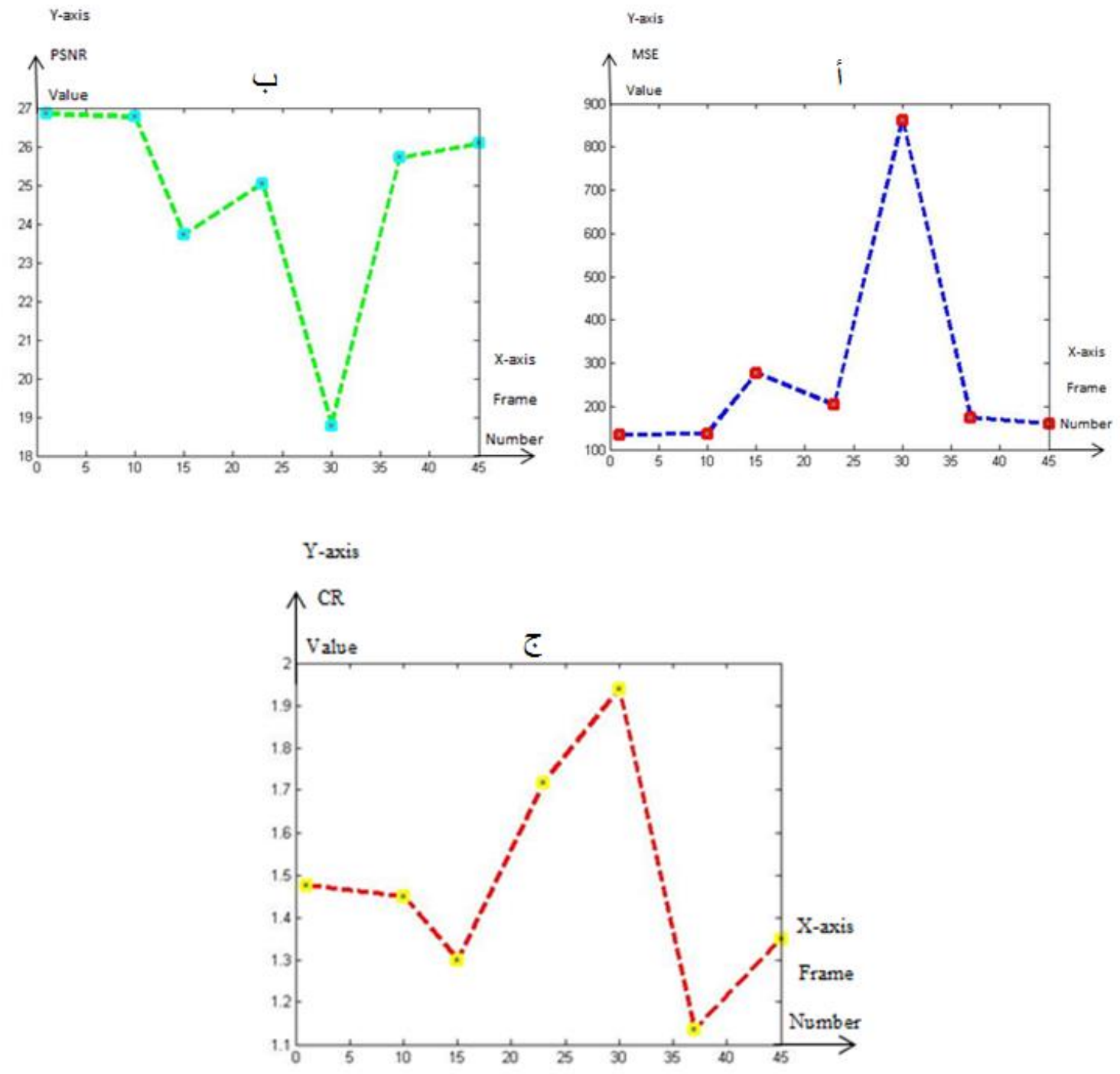

الثكل (3): مقاييس كفاءة عمليه الكبس لمجموعة من العينات (اطر الفيديو)

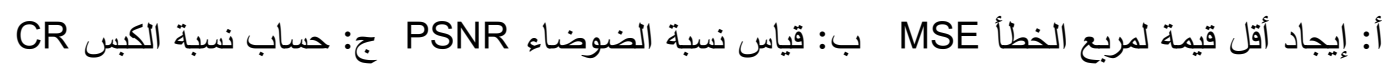
تم العمل على أنموذج آخر من ملفات الفيديو الرقمي للحصول على بلى بيانات ومعلومات مقاييس الكفاءة

والدقة، وكما في الجدول (2).

\begin{tabular}{|c|c|c|c|c|c|c|c|}
\hline$\frac{\text { Frame }}{\text { Number }}$ & $\underline{\text { Rank }}$ & $\underline{\text { Block }}$ & $\begin{array}{c}\text { Frame } \\
\text { Dimension }\end{array}$ & $\underline{\text { PSNR }}$ & $\underline{\text { MSE }}$ & $\underline{\text { Size }(\boldsymbol{k})}$ & $\underline{\text { Compression }}$ \\
\hline $\boldsymbol{I}$ & 2 & 64 & $256^{*} 256$ & 25.8733 & 504.5126 & 12 & $\underline{\text { Ratio }}$ \\
\hline $\boldsymbol{I}$ & 6 & 64 & $256^{*} 256$ & 32.4230 & 111.6608 & 36 & 5.3333 \\
\hline $\boldsymbol{I}$ & 10 & 64 & $256^{*} 256$ & 35.9397 & 49.6858 & 60 & 3.2000 \\
\hline $\boldsymbol{I}$ & 30 & 64 & $256^{*} 256$ & 52.0994 & 1.2030 & 180 & 1.0667 \\
\hline $\boldsymbol{I}$ & 30 & 128 & $256^{*} 256$ & 39.5266 & 21.7540 & 90 & 2.1333 \\
\hline $\boldsymbol{I}$ & 30 & 256 & $256^{*} 256$ & 33.7097 & 83.0295 & 45 & 4.2667 \\
\hline $\mathbf{1 0 0}$ & 2 & 64 & $256^{*} 256$ & 26.5503 & 431.6891 & 12 & 16 \\
\hline $\mathbf{1 0 0}$ & 6 & 64 & $256^{*} 256$ & 32.4932 & 109.8714 & 36 & 5.3333 \\
\hline $\mathbf{1 0 0}$ & 10 & 64 & $256^{*} 256$ & 36.5388 & 43.2835 & 60 & 3.2000 \\
\hline
\end{tabular}




\begin{tabular}{|c|c|c|c|c|c|c|c|}
\hline \multicolumn{100}{|c|}{} & 30 & 64 & $256^{*} 256$ & 54.1215 & 0.7552 & 180 & 1.0667 \\
\hline $\mathbf{1 0 0}$ & 30 & 128 & $256^{*} 256$ & 41.3333 & 14.3505 & 90 & 2.1333 \\
\hline $\mathbf{1 0 0}$ & 30 & 256 & $256^{*} 256$ & 35.5964 & 53.7721 & 45 & 4.2667 \\
\hline $\mathbf{1 5 0}$ & 2 & 64 & $256^{*} 256$ & 27.9064 & 315.9057 & 12 & 16 \\
\hline $\mathbf{1 5 0}$ & 6 & 64 & $256^{*} 256$ & 32.7659 & 103.1828 & 36 & 5.3333 \\
\hline $\mathbf{1 5 0}$ & 10 & 64 & $256^{*} 256$ & 35.7001 & 52.5043 & 60 & 3.2000 \\
\hline $\mathbf{1 5 0}$ & 30 & 64 & $256^{*} 256$ & 49.6750 & 2.1023 & 180 & 1.0667 \\
\hline $\mathbf{1 5 0}$ & 30 & 128 & $256^{*} 256$ & 38.6208 & 26.7994 & 90 & 2.1333 \\
\hline $\mathbf{1 5 0}$ & 30 & 256 & $256^{*} 256$ & 33.7526 & 82.2136 & 45 & 4.2667 \\
\hline $\mathbf{2 0 0}$ & 2 & 64 & $256^{*} 256$ & 25.1860 & 591.0157 & 12 & 16 \\
\hline $\mathbf{2 0 0}$ & 6 & 64 & $256^{*} 256$ & 32.7119 & 104.4744 & 36 & 5.3333 \\
\hline $\mathbf{2 0 0}$ & 10 & 64 & $256^{*} 256$ & 36.9670 & 39.2197 & 60 & 3.2000 \\
\hline $\mathbf{2 0 0}$ & 30 & 64 & $256^{*} 256$ & 52.3848 & 1.1265 & 180 & 1.0667 \\
\hline $\mathbf{2 0 0}$ & 30 & 128 & $256^{*} 256$ & 42.8381 & 10.1483 & 90 & 2.1333 \\
\hline $\mathbf{2 0 0}$ & 30 & 256 & $256^{*} 256$ & 34.6385 & 67.0433 & 45 & 4.2667 \\
\hline $\mathbf{2 7 0}$ & 2 & 64 & $256^{*} 256$ & 23.4642 & 878.5830 & 12 & 16 \\
\hline $\mathbf{2 7 0}$ & 6 & 64 & $256^{*} 256$ & 29.0997 & 240.0126 & 36 & 5.3333 \\
\hline $\mathbf{2 7 0}$ & 10 & 64 & $256^{*} 256$ & 32.3381 & 113.8653 & 60 & 3.2000 \\
\hline $\mathbf{2 7 0}$ & 30 & 64 & $256^{*} 256$ & 44.7164 & 6.5851 & 180 & 1.0667 \\
\hline $\mathbf{2 7 0}$ & 30 & 128 & $256^{*} 256$ & 35.4730 & 55.3224 & 90 & 2.1333 \\
\hline $\mathbf{2 7 0}$ & 30 & 256 & $256^{*} 256$ & 30.2237 & 185.2821 & 45 & 4.2667 \\
\hline
\end{tabular}

الجدول(2): بيانات الأطر المكبوسة لعينات من أطر الفيديو

من خلال الجدول(2) تم الحصول على بيانات طبقت على عينات من أطر مختلفة من الفيديو الرقمي المدخل، فتم التعامل مع عن الإن

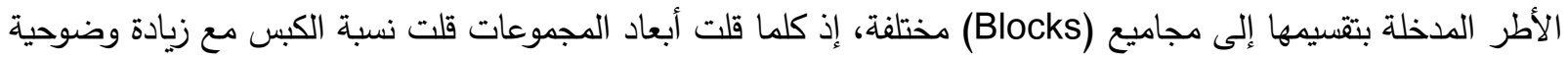
الإطار الناتج، واعتمد على قيمة الرتبة (Rank) والعمل على قيم مختلفة منها، والثكل (4) يوضح وضوحية الأطر المكبوسة وجودتها حسب قيم الرتبة وتقسيم المجاميع.

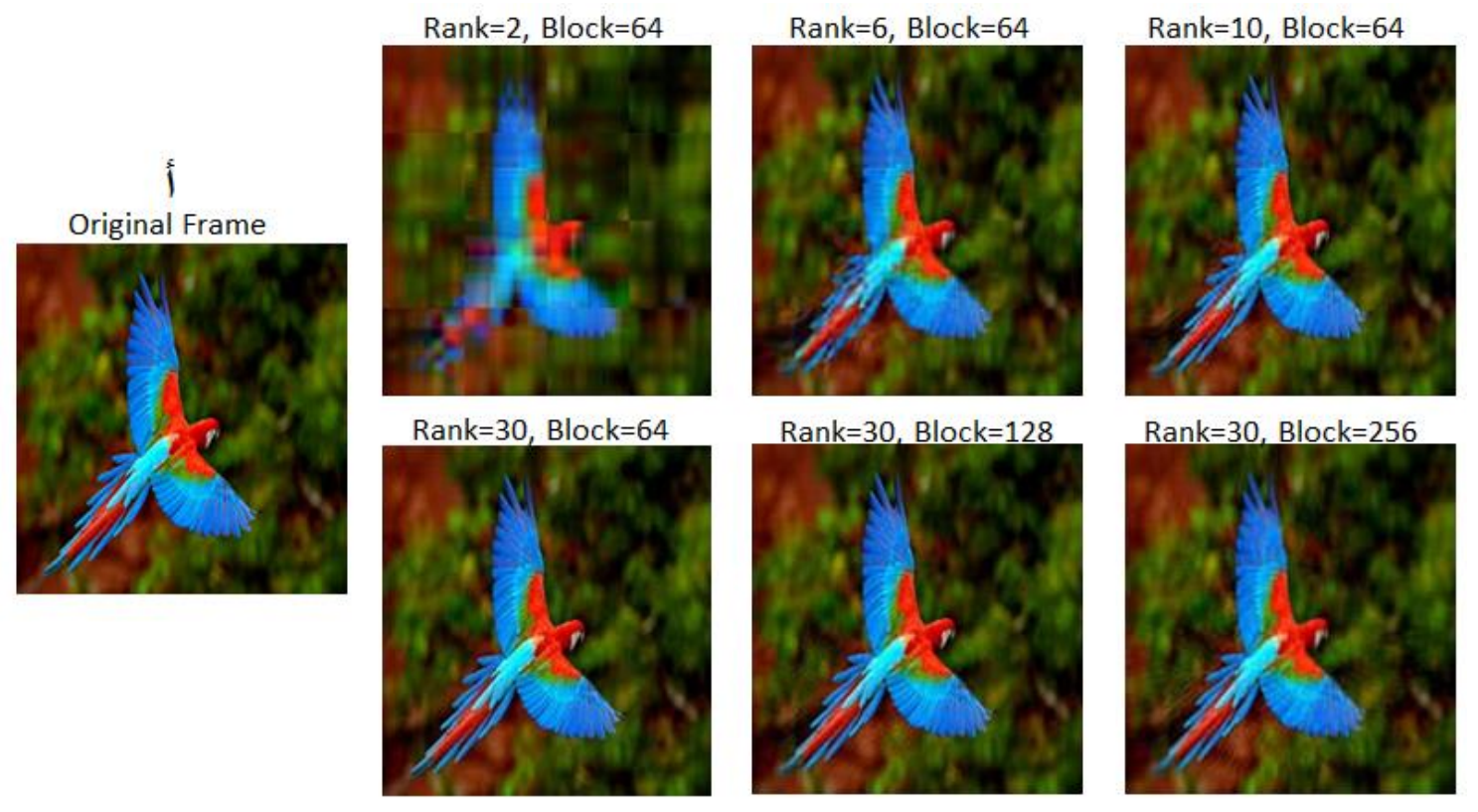




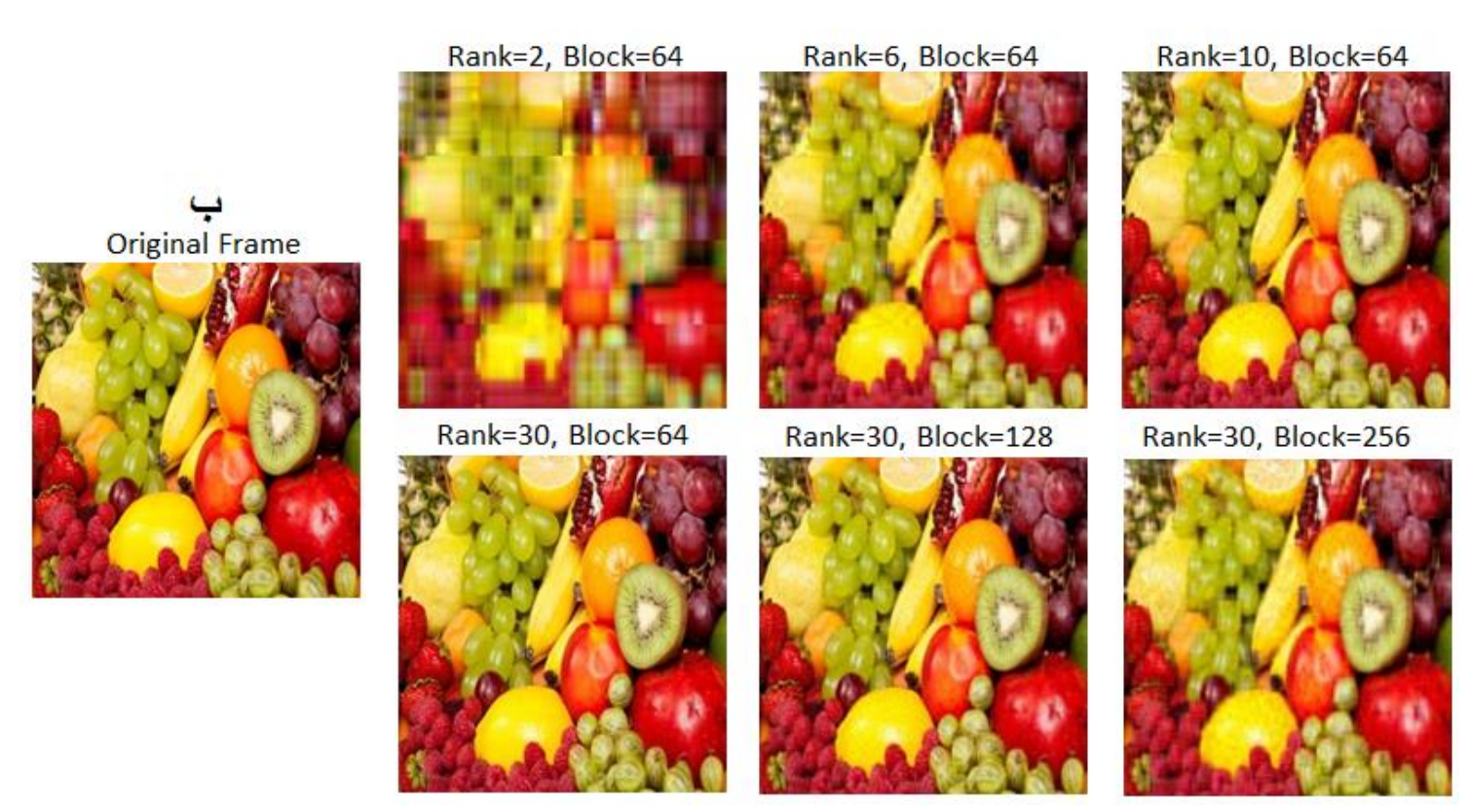

الثكل (4): وضوحية الأطر المكبوسة وجودتها حسب قيم الرتبة وتقسيم المجاميع إذ:

أ-تطبيق عملية الكبس على الإطار رقم 100 ب-تطبيق عملية الكبس على الإطار رقم 270

\section{Conclusions الاستنتاجات}

من خلال تطبيق خوارزميات العمل المقترحة لكبس ملفات القيديو الرقمي باستخدام تقانة تحليل القيمة

$$
\text { الأحادية (SVD)، تم التوصل إلى الاستتتاجات الآتية: }
$$

1-أظهرت النتائج أن استخدام تقانة تحليل القيمة الأحادية (SVD) أداة فعالة ومشجعة لكبس ملفات الفيديو الرقمي، إذ طبتت خوارزميات العمل على أكثر من مقطع فيديو رقمي، وتعد تقانة تحليل القيمة الأحادية كفوهة

$$
\text { وتعطي نتائج جيدة ويمكن الحصول على بيانات قريبة من البيانات الأصلية. }
$$

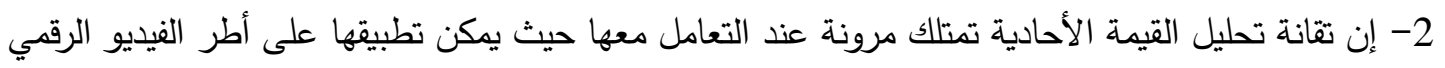

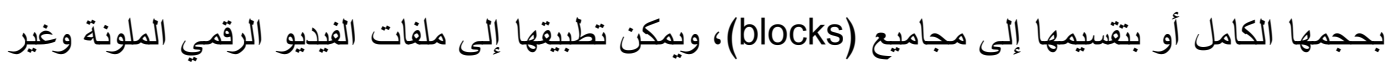

$$
\text { الملونة وبأحجام مختلفة. }
$$

3- تعد عملية الكبس بتقانة تحليل القيمة الأحادية بفقدان، وذلك لفقدان جزء من البيانات بعد إجراء عملية

$$
\text { الكبس. }
$$

4- تعد تقانة تحليل القيمة الأحادية من تقانات الجبر الخطي وتمثل طريقة كبس عالية المستوى تمكن المستخدم

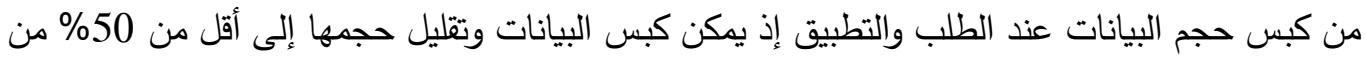

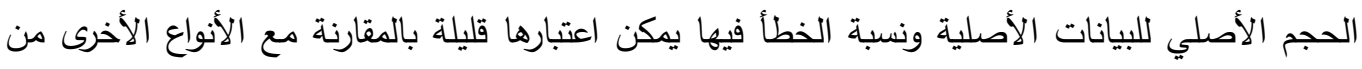

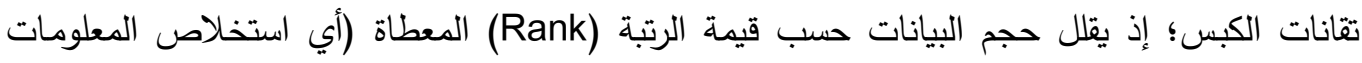
المهمة من البيانات) وخزنها بدون أن تشغل مساحة كبيرة وبهذا يمكن الحصول على بيانات لا تختلف كثيراً 
5- إن جودة الكبس ودقة البيانات تعتمد على قيمة الرتبة (Rank)؛ إذ كلما كانت قيمة الرتبة قليلة كانت نسبة الكبس كبيرة ولكن دقة البيانات المسترجعة ووضوحها قليل، وإذا كانت قيمة الرتبة كبيرة كانت نسبة الكبس قليلة ولكن دقة الصورة المسترجعة ووضوحها كبير • 6- إن نسبة الكبس تختلف من إطار إلى آخر بحسب كثافة البيانات داخل كل إطار بحسب النتائج الظاهرة ضمن الجدولين (1) و (2)، فالإطار الذي يحتوي بيانات ومعلومات قليلة تكون نسبة الكبس فيه أقل من

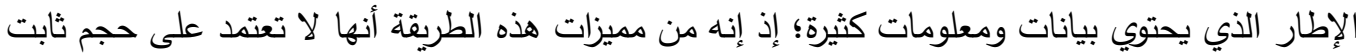
للكبس ولكنها تعتمد على كمية المعلومات المطلوب خزنها وكبسها لغرض استرجاعها أي تعتمد على دقة التفاصيل الموجودة ضمن أطر الفيديو.

\section{Recommendations 7 التوصيات}

إن ما تم بناؤه في هذه الخوارزميات يمكن أن يكون نقطة انطلاق لأفكار وأعمال مستقبلية ذات أهداف مشتركة، وعليه اقترحت الأعمال المستقبلية الآتية:

1-تهجين تقانة تحليل القيمة الأحادية (SVD) مع تقانات كبس أخرى، مثل تقانة التحويل المويجي (Wavelets) وغيرها.

2-تقسيم أطر الفيديو إلى مجاميع وتطبيق تقانة تحليل القيمة الأحادية (SVD) باستخدام رتب (Rank) مختلفة

$$
\text { لعمل مجموعة حسب شروط وقواعد معينة. }
$$

3-إمكانية استخدام تقانات الذكاء الاصطناعي مع تقانة تحليل القيمة الأحادية مثل الثبكات العصبية الاصطناعية

$$
\text { وغيرها. }
$$

4- إمكانية العمل مع الزمن الحقيقي (Real Time) في عملية إدخال ومعالجة الفيديو الرقمي التي تستخدم غالباً عبر شبكات الاتصالات لتقليل حجم البيانات المرسلة والمستقبلة. 


\section{المصاد}

[1] Aishwarya K. M., Rachana R., Sobarad M., Vipula S., 2016, "Lossy Image Compression using SVD Coding Algorithm", This full-text paper was peerreviewed and accepted to be presented at the IEEE WiSPNET conference.

[2] Bruno R. H., Auguste R. P., Raft R. N., 2016, "Image Compression with SVD: A New Quality Metric Based On Energy Ratio", IJCSN International Journal of Computer Science and Network, Volume 5, Issue 6, December, ISSN (Online) : 2277-5420.

[3] Gholamreza A., 2017, "Super Resolution Based Image Compression Using Wavelet Transforms and Singular Value Decomposition", Tamap Journal of Engineering, Volume 2017, Article ID 3, Research Article.

[4] Lei Y., 2011, "Image And Video Compression And Copyright Protection", A Dissertation Presented To The Graduate School Of The University Of Florida In Partial Fulfillment Of The Requirements For The Degree Of Doctor Of Philosophy, University Of Florida.

[5] Mahalakshmi R., Mahendran S. K., 2017, "Analysis of Video Compression Technique", International Journal of Advanced Research in Computer Science \& Technology (IJARCST 2017) 52 Vol. 5, Issue 1 (Jan. - Mar.).

[6] Maytham S., Aqeel S., 2017, "Hybrid Speech Compression method Based on DWT and SVD", International Journal of Applied Engineering Research ISSN 0973-4562 Volume 12, Number 21 (2017) pp. 11493-11499.

[7] Mounika K., Lakshmi S. N., Alekya K., 2015, "SVD BASED IMAGE COMPRESSION", International Journal of Engineering Research and General Science Volume 3, Issue 2, March-April, ISSN 2091-2730.

[8] OGE M., 2011, "PRACTICAL IMAGE AND VIDEO PROCESSING USING MATLAB", Copyright (C) by John Wiley \& Sons, Inc. All rights reserved, Published simultaneously in Canada.

[9] Pabi J. A., Puviarasan N., Aruna P., 2017, "Fast Singular value decomposition based image compression using butterfly particle swarm optimization technique (SVD-BPSO)", Volume 4, Issue 4, April, pp. 128135 ISSN (O): 2349-7084 International Journal of Computer Engineering In Research Trends.

[10] Samruddhi K., Reena R., 2013, "Image Compression using Singular Value Decomposition", International Journal of Advancements in Research \& Technology, Volume 2, Issue 8, August-2013 244 ISSN 2278-7763.

[11] Shafik D. S., Chavan M. S., 2017, "Comparative Analysis of Singular Value Decomposition (SVD) and Wavelet Difference Reduction (WDR) based Image Compression", International Journal of Engineering Research and Technology. ISSN 0974-3154 Volume 10, Number 1.

[12] Sik K. C. Y., Berk C., 2017, "Video Compression Using Recurrent Convolutional Neural Networks", Computer Science bcoker@stanford.edu, Electrical Engineering, cedyue@ stanford.edu. 
[13] Swati P., Kumudani S. D., 2017, “An Efficient Image Compression using Singular Value Decomposition with Scale Invariant Feature Transform", International Journal of Computer Applications (0975 - 8887) Volume 159 - No 2, February.

[14] Tekalp A. M., 1995, "Digital Video Processing”, University of Rochester, Prentice Hall PTR, Upper Saddle River, NJ 07458.

[15] Tilaye T., 1997, "Digital Video Compression For Software-based Real-time Applications", A thesis submitted in conformity with the requirements for the degree of Master of Applied Science, Graduate Department of Electrical and Computer Engineering, in the University of Toronto. 